APPLICATIONES MATHEMATICAE

27,4 (2000), pp. $445-454$

W. POPIŃSKI (Warszawa)

\title{
CONVERGENCE RATES OF ORTHOGONAL SERIES REGRESSION ESTIMATORS
}

Abstract. General conditions for convergence rates of nonparametric orthogonal series estimators of the regression function $f(x)=E(Y \mid X=x)$ are considered. The estimators are obtained by the least squares method on the basis of a random observation sample $\left(Y_{i}, X_{i}\right), i=1, \ldots, n$, where $X_{i} \in A \subset \mathbb{R}^{d}$ have marginal distribution with density $\varrho \in L^{1}(A)$ and $\operatorname{Var}(Y \mid X=x)$ is bounded on $A$. Convergence rates of the errors $E_{X}(f(X)-$ $\left.\widehat{f}_{N}(X)\right)^{2}$ and $\left\|f-\widehat{f}_{N}\right\|_{\infty}$ for the estimator $\widehat{f}_{N}(x)=\sum_{k=1}^{N} \widehat{c}_{k} e_{k}(x)$, constructed using an orthonormal system $e_{k}, k=1,2, \ldots$, in $L^{2}(A)$, are obtained.

1. Introduction. Let $\left(Y_{i}, X_{i}\right), i=1, \ldots, n$, be a random sample of size $n$ from the distribution of $(X, Y)$, where $X$ represents a predictor variable and $Y$ a real-valued response variable. We assume that $X$ ranges over a compact subset $A$ of some euclidean space $\mathbb{R}^{d}, d \geq 1$, and has absolutely continuous distribution with density $\varrho \in L^{1}(A)$. Set $f(x)=E(Y \mid X=$ $x), \sigma^{2}(x)=\operatorname{Var}(Y \mid X=x)$ and assume that $\sigma^{2}(x) \leq C$ for $x \in A$ and the function $f$ can be uniformly approximated on this set by finite linear combinations of functions $e_{k}, k=1,2, \ldots$, forming a complete orthonormal system in $L^{2}(A)$. We consider the problem of estimating the regression function $f$ using series estimators of the form

$$
\widehat{f}_{N}(x)=\sum_{k=1}^{N} \widehat{c}_{k N} e_{k}(x),
$$

where the vector of coefficient estimators $\widehat{c}_{N}=\left(\widehat{c}_{1 N}, \ldots, \widehat{c}_{N N}\right)^{T}$ is, for a fixed $N$, obtained by the least squares method, i.e.

2000 Mathematics Subject Classification: 62G08, 62G20.

Key words and phrases: nonparametric series regression, least squares method, orthonormal system, convergence rate. 


$$
\widehat{c}_{N}=\arg \min _{a \in \mathbb{R}^{N}} \frac{1}{n} \sum_{i=1}^{n}\left(Y_{i}-\left\langle a, e^{N}\left(X_{i}\right)\right\rangle\right)^{2},
$$

where $e^{N}(x)=\left(e_{1}(x), \ldots, e_{N}(x)\right)^{T}$.

The vector $\widehat{c}_{N}$ can be obtained as a solution of the normal equations

$$
G_{n} \widehat{c}_{N}=g_{n}
$$

where

$$
G_{n}=\frac{1}{n} \sum_{i=1}^{n} e^{N}\left(X_{i}\right) e^{N}\left(X_{i}\right)^{T}, \quad g_{n}=\frac{1}{n} \sum_{i=1}^{n} Y_{i} e^{N}\left(X_{i}\right),
$$

and when $\operatorname{det} G_{n} \neq 0$ it is uniquely determined. Set $G(N)=E G_{n}$ and observe that for any vector $v=\left(v_{1}, \ldots, v_{N}\right)^{T} \in \mathbb{R}^{N}$ the following equality is true:

$$
\begin{aligned}
\langle G(N) v, v\rangle & =\left\langle E e^{N}(X) e^{N}(X)^{T} v, v\right\rangle=\sum_{k=1}^{N} \sum_{l=1}^{N} v_{k} v_{l} \int_{A} e_{k}(x) e_{l}(x) \varrho(x) d x \\
& =\int_{A}\left(\sum_{k=1}^{N} v_{k} e_{k}(x)\right)^{2} \varrho(x) d x .
\end{aligned}
$$

If we assume that $\varrho \geq c>0$, then in view of orthogonality of the functions $e_{k}, k=1,2, \ldots$, the above equality implies that $\lambda(N) \geq c$, where $\lambda(N)$ denotes the minimal eigenvalue of the matrix $G(N)$, and consequently $G(N)$ is nonsingular.

Let us also note that in the case when $\operatorname{det} G_{n} \neq 0$ the estimator $\widehat{f}_{N}$ is invariant under nonsingular linear transformations of $e^{N}(x)$, i.e. it does not change when we use the vector function $h^{N}(x)=B e^{N}(x)$, where $B$ is a nonsingular matrix, instead of $e^{N}(x)$ for constructing it. In consequence, in the case when $\varrho \geq c>0$ and $\operatorname{det} G_{n} \neq 0$ the series estimator considered can be represented in the form $\widehat{f}_{N}(x)=\left\langle h^{N}(x), \widehat{b}_{N}\right\rangle$, where $h^{N}(x)=G(N)^{-1 / 2} e^{N}(x)$ and the vector of coefficient estimators $\widehat{b}_{N}=$ $G(N)^{1 / 2} \widehat{c}_{N}$ is determined by the least squares method. Such a representation of $\widehat{f}_{N}$ is convenient, since then $E h^{N}(X) h^{N}(X)^{T}=I_{N}$ and consequently if $h^{N}(x)=\left(h_{1 N}(x), \ldots, h_{N N}(x)\right)^{T}$, then the functions $h_{k N}(x), k=1, \ldots, N$, are orthonormal with weight $\varrho$, i.e. $\int_{A} h_{k N}(x) h_{l N}(x) \varrho(x) d x=\delta_{k l}$.

Moreover, if $H_{n}=(1 / n) \sum_{i=1}^{n} h^{N}\left(X_{i}\right) h^{N}\left(X_{i}\right)^{T}$ is the matrix of normal equations corresponding to the vector function $h^{N}(x)$, then

$$
\begin{aligned}
E\left\|H_{n}-I\right\|^{2} & =\sum_{k=1}^{N} \sum_{l=1}^{N} E\left(\frac{1}{n} \sum_{i=1}^{n} h_{k N}\left(X_{i}\right) h_{l N}\left(X_{i}\right)-\delta_{k l}\right)^{2} \\
& \leq \sum_{k=1}^{N} \sum_{l=1}^{N} \frac{1}{n} E h_{k N}^{2}(X) h_{l N}^{2}(X)=\frac{1}{n} E\left\|h^{N}(X)\right\|^{2}\left\|h^{N}(X)\right\|^{2}
\end{aligned}
$$




$$
\leq \frac{1}{n}\left\|h^{N}\right\|_{\infty}^{2} E\left\|h^{N}(X)\right\|^{2}=\frac{N}{n}\left\|h^{N}\right\|_{\infty}^{2},
$$

where $\left\|h^{N}\right\|_{\infty}=\sup _{s \in A}\left\|h^{N}(s)\right\|$, and since $h^{N}(x)=G(N)^{-1 / 2} e^{N}(x)$ we also have

so finally

$$
\left\|h^{N}\right\|_{\infty}^{2} \leq \lambda(N)^{-1}\left\|e^{N}\right\|_{\infty}^{2} \leq\left\|e^{N}\right\|_{\infty}^{2} c^{-1}
$$

$$
E\left\|H_{n}-I\right\|^{2} \leq \frac{N}{c n}\left\|e^{N}\right\|_{\infty}^{2}
$$

If we put $M_{N}=\left\|e^{N}\right\|_{\infty}$ and $\lambda_{n}$ denotes the smallest eigenvalue of the matrix $H_{n}$, then since $\left|\lambda_{n}-1\right| \leq\left\|H_{n}-I\right\|$, we also have

$$
E\left|\lambda_{n}-1\right|^{2} \leq \frac{N M_{N}^{2}}{c n},
$$

which implies that $\lambda_{n} \rightarrow 1$ in probability on condition that $N M_{N}^{2} / n \rightarrow 0$ as $n \rightarrow \infty$. This fact will be used to prove the results presented below. Let us also note that for $\varrho \geq c>0$, by (3) we have $P\left(\operatorname{det} G_{n}=0\right)=P\left(\operatorname{det} H_{n}\right.$ $=0) \leq P\left(\lambda_{n}<1 / 2\right) \leq 4 N M_{N}^{2} c^{-1} / n$. Thus, the conditions $\varrho \geq c>0$ and $N M_{N}^{2} / n \rightarrow 0$ as $n \rightarrow \infty$ assure that $P\left(\operatorname{det} G_{n}=0\right) \rightarrow 0$, i.e. the estimator is uniquely determined with growing probability.

In this work we continue the investigations of [8]-[10] on asymptotic properties of series regression estimators by considering convergence rates of the errors $E_{X}\left(f(X)-\widehat{f}_{N}(X)\right)^{2}$ and $\left\|f-\widehat{f}_{N}\right\|_{\infty}$. We give sufficient conditions for the convergence rates and extend the results of [9], [10] where only convergence in probability of such errors for trigonometric and polynomial estimators was investigated. Huang [4] has recently obtained general conditions for convergence rates of $E_{X}\left(f(X)-\widehat{f}_{N}(X)\right)^{2}$ for the relevant series estimators, under the assumption that $D \geq \varrho \geq c>0$, but the estimator measurability and uniqueness conditions are not discussed there. In the present work it is shown that the boundedness condition imposed on the density $\varrho$ can be relaxed.

Asymptotic properties of other nonparametric series regression estimators for similar observation models were investigated in the works of Lugosi and Zeger [6] and Györfi and Walk [3] but the results obtained there concern the universal consistency of their estimators and the problem of convergence rates is only briefly discussed in [6]. The series estimator considered in [6] is obtained via constrained empirical risk minimization (whereas our estimators are obtained by unconstrained empirical risk minimization) and the one considered in [3] is based on stochastic approximation procedure in a function space. Convergence rates of $E_{X}\left(f(X)-\widetilde{f}_{N}(X)\right)^{2}$ for estimators $\widetilde{f}_{N}$ constructed using radial basis functions and neural networks are investigated in [7]. Properties of series estimators in the case of other observation models are investigated in [13]. 
2. Convergence rates of the $L^{2}$-error. Let $\chi_{n}$ denote the indicator function of $\left\{\lambda_{n} \geq 1 / 2\right\}$. According to (3) we have $P\left(\lambda_{n}<1 / 2\right) \leq$ $4 N M_{N}^{2} c^{-1} / n$ and the assumption $N M_{N}^{2} / n \rightarrow 0$ as $n \rightarrow \infty$ implies that $P\left(\chi_{n} \neq 1\right) \rightarrow 0$.

We need the following lemmas. Only the proof of the second lemma is given since the first one is proved in [11].

LEMMA 2.1. Let $v=\left(v_{1}, \ldots, v_{n}\right)^{T} \in \mathbb{R}^{n}$ and

$$
H_{n}=\frac{1}{n} \sum_{i=1}^{n} h^{N}\left(X_{i}\right) h^{N}\left(X_{i}\right)^{T}
$$

Then

$$
\frac{1}{n^{2}} \sum_{i=1}^{n} \sum_{j=1}^{n} v_{i} v_{j} h^{N}\left(X_{i}\right)^{T} H_{n}^{-1} h^{N}\left(X_{j}\right) \leq \frac{1}{n} \sum_{i=1}^{n} v_{i}^{2} .
$$

Lemma 2.2. Assume that $\varrho \geq c>0$ and for $N>0$ there exist $f_{N} \in$ $\operatorname{span}\left\{e_{1}, \ldots, e_{N}\right\}$ such that $\left\|f-f_{N}\right\|_{\infty}=O\left(N^{-\alpha}\right)$ as $N \rightarrow \infty$, where $\alpha>0$. If $f_{N}(x)=\left\langle h^{N}(x), b_{N}\right\rangle$ and $\widehat{b}_{N}=H_{n}^{-1} h_{n}$, where $h_{n}=(1 / n) \sum_{i=1}^{n} Y_{i} h^{N}\left(X_{i}\right)$, then

$$
E \chi_{n}\left\|\widehat{b}_{N}-b_{N}\right\|^{2}=O\left(N / n+N^{-2 \alpha}\right) .
$$

Proof. Putting $\eta_{i}=Y_{i}-f\left(X_{i}\right), i=1, \ldots, n$, and $f(x)=f_{N}(x)+r_{N}(x)$, we have

$$
\text { (4) } \widehat{b}_{N}=b_{N}+H_{n}^{-1}\left(\frac{1}{n} \sum_{i=1}^{n} r_{N}\left(X_{i}\right) h^{N}\left(X_{i}\right)\right)+H_{n}^{-1}\left(\frac{1}{n} \sum_{i=1}^{n} \eta_{i} h^{N}\left(X_{i}\right)\right) \text {. }
$$

Now, putting $D_{n}=\left(X_{1}, \ldots, X_{n}\right)$, since $\operatorname{Var}\left(Y \mid X=X_{i}\right) \leq C, i=1, \ldots, n$, we easily obtain

$$
\begin{aligned}
E\left[\chi_{n}\left\|H_{n}^{-1 / 2} \frac{1}{n} \sum_{i=1}^{n} \eta_{i} h^{N}\left(X_{i}\right)\right\|^{2} \mid D_{n}\right] \\
=\chi_{n} E\left[\frac{1}{n^{2}} \sum_{i=1}^{n} \sum_{j=1}^{n} \eta_{i} \eta_{j} h^{N}\left(X_{i}\right)^{T} H_{n}^{-1} h^{N}\left(X_{j}\right) \mid D_{n}\right] \\
\leq \chi_{n} \frac{C}{n^{2}} \sum_{i=1}^{n} h^{N}\left(X_{i}\right)^{T} H_{n}^{-1} h^{N}\left(X_{i}\right) \\
=\chi_{n} \frac{C}{n^{2}} \operatorname{Tr}\left(\sum_{i=1}^{n} h^{N}\left(X_{i}\right) h^{N}\left(X_{i}\right)^{T} H_{n}^{-1}\right) \leq \frac{C N}{n}
\end{aligned}
$$

The last inequality implies

$$
E \chi_{n}\left\|H_{n}^{-1 / 2} \frac{1}{n} \sum_{i=1}^{n} \eta_{i} h^{N}\left(X_{i}\right)\right\|^{2} \leq C N / n
$$


which further yields

$$
\begin{aligned}
E \chi_{n} \| H_{n}^{-1} & \frac{1}{n} \sum_{i=1}^{n} \eta_{i} h^{N}\left(X_{i}\right) \|^{2} \\
& =E \chi_{n}\left(\frac{1}{n^{2}} \sum_{i=1}^{n} \eta_{i} h^{N}\left(X_{i}\right)^{T} H_{n}^{-1 / 2} H_{n}^{-1} H_{n}^{-1 / 2} \sum_{j=1}^{n} \eta_{j} h^{N}\left(X_{j}\right)\right) \\
& \leq E \chi_{n} \lambda_{n}^{-1}\left\|H_{n}^{-1 / 2} \frac{1}{n} \sum_{i=1}^{n} \eta_{i} h^{N}\left(X_{i}\right)\right\|^{2} \leq 2 C N / n
\end{aligned}
$$

since $\chi_{n} \lambda_{n}^{-1} \leq 2$. In view of Lemma 2.1 it is also easy to see that

$$
\begin{aligned}
E \chi_{n} \| & H_{n}^{-1} \frac{1}{n} \sum_{i=1}^{n} r_{N}\left(X_{i}\right) h^{N}\left(X_{i}\right) \|^{2} \\
& =E \chi_{n}\left(\frac{1}{n^{2}} \sum_{i=1}^{n} r_{N}\left(X_{i}\right) h^{N}\left(X_{i}\right)^{T} H_{n}^{-1 / 2} H_{n}^{-1} H_{n}^{-1 / 2} \sum_{j=1}^{n} r_{N}\left(X_{j}\right) h^{N}\left(X_{j}\right)\right) \\
& \leq E \chi_{n} \lambda_{n}^{-1}\left(\frac{1}{n^{2}} \sum_{i=1}^{n} r_{N}\left(X_{i}\right) h^{N}\left(X_{i}\right)^{T} H_{n}^{-1} \sum_{j=1}^{n} r_{N}\left(X_{j}\right) h^{N}\left(X_{j}\right)\right) \\
& \leq E \chi_{n} \lambda_{n}^{-1}\left(\frac{1}{n} \sum_{i=1}^{n} r_{N}^{2}\left(X_{i}\right)\right) \leq E \chi_{n} \lambda_{n}^{-1} \max _{1 \leq i \leq n}\left|r_{N}^{2}\left(X_{i}\right)\right|=O\left(N^{-2 \alpha}\right) .
\end{aligned}
$$

The above bounds together with (4) imply the assertion of the lemma.

In the case when the regression function $f$ is square-integrable and the density $\varrho$ satisfies the additional condition $D \geq \varrho \geq c>0$ we also have the following lemma.

Lemma 2.3. Assume that $D \geq \varrho \geq c>0, f \in L^{2}(A)$ and $f_{N}(x)=$ $\left\langle h^{N}(x), b_{N}\right\rangle$ is its orthogonal projection on $\operatorname{span}\left\{e_{1}, \ldots, e_{N}\right\}$. If $\widehat{b}_{N}=$ $H_{n}^{-1} h_{n}$, then

$$
E \chi_{n}\left\|\widehat{b}_{N}-b_{N}\right\|^{2}=O\left(N / n+\left\|f-f_{N}\right\|^{2}\right) .
$$

Proof. First observe that for $r_{N}=f-f_{N}$,

$$
E\left(\frac{1}{n} \sum_{i=1}^{n} r_{N}^{2}\left(X_{i}\right)\right)=E r_{N}^{2}(X) \leq D\left\|f-f_{N}\right\|^{2},
$$

and then follow the proof of Lemma 2.2.

Now, we can prove the following theorem on convergence rates of the estimators considered. 
Theorem 2.1. Assume that $\varrho \geq c>0$, the sequence of natural numbers $N(n), n=1,2, \ldots$, satisfies

$$
\lim _{n \rightarrow \infty} N(n)=\infty, \quad \lim _{n \rightarrow \infty} \frac{N(n) M_{N(n)}^{2}}{n}=0,
$$

and for $N>0$ there exist $f_{N} \in \operatorname{span}\left\{e_{1}, \ldots, e_{N}\right\}$ such that $\left\|f-f_{N}\right\|_{\infty}=$ $O\left(N^{-\alpha}\right)$ as $N \rightarrow \infty$, where $\alpha>0$. Then the orthogonal series estimator $\widehat{f}_{N(n)}$ satisfies

$$
\int_{A}\left(f-\widehat{f}_{N(n)}\right)^{2} \varrho=O_{p}\left(N(n) / n+N(n)^{-2 \alpha}\right) .
$$

Proof. Putting $f_{N}(x)=\left\langle h^{N}(x), b_{N}\right\rangle$, we easily obtain, by the triangle inequality and the equality $E h^{N}(X) h^{N}(X)^{T}=I_{N}$,

$$
\begin{aligned}
\chi_{n} \int_{A}\left(f-\widehat{f}_{N}\right)^{2} \varrho & =\chi_{n} \int_{A}\left(f(x)-f_{N}(x)+\left\langle h^{N}(x), b_{N}-\widehat{b}_{N}\right\rangle\right)^{2} \varrho(x) d x \\
& \leq 2 \chi_{n} \int_{A}\left(f(x)-f_{N}(x)\right)^{2} \varrho(x) d x+2 \chi_{n}\left\|b_{N}-\widehat{b}_{N}\right\|^{2} \\
& \leq O\left(N^{-2 \alpha}\right)+2 \chi_{n}\left\|b_{N}-\widehat{b}_{N}\right\|^{2} .
\end{aligned}
$$

As remarked earlier, the assumption $N(n) M_{N(n)}^{2} / n \rightarrow 0$ assures that $P\left(\chi_{n} \neq 1\right) \rightarrow 0$ so the assertion follows by applying Lemma 2.2.

In the case when the regression function can be approximated in the mean-square sense the following theorem holds.

Theorem 2.2. Assume that $D \geq \varrho \geq c>0$, the sequence of natural numbers $N(n), n=1,2, \ldots$, satisfies

$$
\lim _{n \rightarrow \infty} N(n)=\infty, \quad \lim _{n \rightarrow \infty} \frac{N(n) M_{N(n)}^{2}}{n}=0,
$$

and $f_{N}$ is the orthogonal projection of the regression function $f \in L^{2}(A)$ on $\operatorname{span}\left\{e_{1}, \ldots, e_{N}\right\}$. Then the orthogonal series estimator $\widehat{f}_{N(n)}$ satisfies

$$
\int_{A}\left(f-\widehat{f}_{N(n)}\right)^{2} \varrho=O_{p}\left(N(n) / n+\left\|f-f_{N(n)}\right\|^{2}\right) .
$$

P r o of. Observe that $\int_{A}\left(f-f_{N}\right)^{2} \varrho \leq D\left\|f-f_{N}\right\|^{2}$ and follow the proof of Theorem 2.1 using Lemma 2.3 instead of Lemma 2.2. In fact we can even prove that $E \chi_{n} \int_{A}\left(f-\widehat{f}_{N(n)}\right)^{2} \varrho=O\left(N(n) / n+\left\|f-f_{N(n)}\right\|^{2}\right)$.

Since for $f \in L^{2}(A)$ we have $\left\|f-f_{N}\right\|^{2} \rightarrow 0$ as $N \rightarrow \infty$, under the assumptions of Theorem 2.2, $E_{X}\left(f(X)-\widehat{f}_{N(n)}(X)\right)^{2}=o_{p}(1)$ and consequently also $\left\|f-\widehat{f}_{N(n)}\right\|^{2}=o_{p}(1)$. Moreover, if $\left\|f-f_{N}\right\|=O\left(N^{-\alpha}\right)$, where $\alpha>0$, Theorem 2.2 allows one to obtain convergence rates of the error 
$E_{X}\left(f(X)-\widehat{f}_{N(n)}(X)\right)^{2}$. The term $N / n$ in the above formulae for convergence rates essentially corresponds to a variance term, and $N^{-2 \alpha}$ to a bias term. When $N(n)$ is chosen so that these two terms go to zero at the same rate, which occurs when $N(n) \sim n^{1 /(1+2 \alpha)}$ (i.e. $r_{1} \geq N(n) n^{-1 /(1+2 \alpha)} \geq r_{2}$, $\left.r_{1}, r_{2}>0\right)$, the convergence rate of the estimators will be $n^{-2 \alpha /(1+2 \alpha)}$. Thus, Stone's [12] bound on the best obtainable rate is attained.

Let us also remark that the error $E_{X}\left(f(X)-\widehat{f}_{N}(X)\right)^{2}$, for which convergence rates were obtained above, is related to the prediction error $E(Y-$ $\left.\widehat{f}_{N}(X)\right)^{2}=E_{X}\left(f(X)-\widehat{f}_{N}(X)\right)^{2}+E \sigma^{2}(X)$.

It is easy to see that for the estimator $\widetilde{f}_{N}$ defined by the formula

$$
\widetilde{f}_{N}(x)= \begin{cases}\widehat{f}_{N}(x) & \text { if } \lambda_{n} \geq 1 / 2 \\ 0 & \text { otherwise }\end{cases}
$$

Theorems 2.1 and 2.2 are true, but we also have the following result concerning its IMSE.

Theorem 2.3. Assume that $D \geq \varrho \geq c>0$, the sequence of natural numbers $N(n), n=1,2, \ldots$, satisfies

$$
\lim _{n \rightarrow \infty} N(n)=\infty, \quad \lim _{n \rightarrow \infty} \frac{N(n) M_{N(n)}^{2}}{n}=0,
$$

and $f_{N}$ is the orthogonal projection of the regression function $f \in L^{2}(A)$ on $\operatorname{span}\left\{e_{1}, \ldots, e_{N}\right\}$. Then the orthogonal series estimator $\widetilde{f}_{N(n)}$ satisfies

$$
E \int_{A}\left(f-\widetilde{f}_{N(n)}\right)^{2}=O\left(N(n) / n+\left\|f-f_{N(n)}\right\|^{2}+N(n) M_{N(n)}^{2} / n\right) .
$$

Pr o of. According to the definition of $\widetilde{f}_{N}$, we have

$$
\begin{aligned}
E \int_{A}\left(f-\widetilde{f}_{N}\right)^{2} & =E \chi_{n} \int_{A}\left(f-\widehat{f}_{N}\right)^{2}+E\left(1-\chi_{n}\right) \int_{A} f^{2} \\
& \leq c^{-1} E \chi_{n} \int_{A}\left(f-\widehat{f}_{N}\right)^{2} \varrho+P\left(\lambda_{n}<1 / 2\right)\|f\|^{2} .
\end{aligned}
$$

As remarked in the proof of Theorem 2.2, the first term on the right hand side is $O\left(N(n) / n+\left\|f-f_{N(n)}\right\|^{2}\right)$ and the second term is bounded by $4\|f\|^{2} N M_{N}^{2} c^{-1 / n}$, which completes the proof.

Since $\lambda_{n}$ is the minimal eigenvalue of the matrix $H_{n}$ which is not used in computations we cannot verify directly whether the condition $\lambda_{n} \geq 1 / 2$ is satisfied. However, Theorem 2.3 allows us to learn about IMSE convergence rates of the estimator. Namely, for many orthonormal systems it is possible to obtain a bound of the form

$$
M_{N}^{2}=\left\|e^{N}\right\|_{\infty}^{2}=\sup _{x \in A} \sum_{k=1}^{N} e_{k}^{2}(x) \leq K N,
$$


where $K$ is a constant [1]. This clearly holds for uniformly bounded systems (e.g. the trigonometric system in $L^{2}\left([0,2 \pi]^{d}\right)$ ) but also for strongly localized systems (e.g. the splines, piecewise polynomials) and leveled localized systems (e.g. compactly supported wavelets in $L^{2}[0,1]$ ) [1], [13]. For such systems the condition $N(n) M_{N(n)}^{2} / n \rightarrow 0$ is satisfied if $N(n)^{2} / n \rightarrow 0$ as $n \rightarrow \infty$ and under the assumptions of Theorem 2.3 we have $E\left\|f-\widetilde{f}_{N(n)}\right\|^{2}=$ $O\left(\left\|f-f_{N(n)}\right\|^{2}+N(n)^{2} / n\right)$. Assuming further that $\left\|f-f_{N}\right\|=O\left(N^{-\alpha}\right)$ we see that for $N(n) \sim n^{1 /(2+2 \alpha)}$ the IMSE convergence rate of $\widetilde{f}_{N}$ is $n^{-\alpha /(\alpha+1)}$.

3. Uniform convergence rates. In this section a result on uniform convergence rates of orthogonal series estimators is proved. It extends the results of [9] where only uniform pointwise consistency of trigonometric and polynomial estimators was examined.

Theorem 3.1. Assume that $\varrho \geq c>0$, the sequence of natural numbers $N(n), n=1,2, \ldots$, satisfies

$$
\lim _{n \rightarrow \infty} N(n)=\infty, \quad \lim _{n \rightarrow \infty} \frac{N(n) M_{N(n)}^{2}}{n}=0,
$$

and for $N>0$ there exist $f_{N} \in \operatorname{span}\left\{e_{1}, \ldots, e_{N}\right\}$ such that $\left\|f-f_{N}\right\|_{\infty}=$ $O\left(N^{-\alpha}\right)$ as $N \rightarrow \infty$, where $\alpha>0$. Then the orthogonal series estimator $\widehat{f}_{N(n)}$ satisfies

$$
\left\|f-\widehat{f}_{N(n)}\right\|_{\infty}=O_{p}\left(M_{N(n)}\left(N(n)^{1 / 2} / n^{1 / 2}+N(n)^{-\alpha}\right)\right) .
$$

Proof. Putting $f_{N}(x)=\left\langle h^{N}(x), b_{N}\right\rangle$, by the triangle and Cauchy inequalities we have

$$
\begin{aligned}
\chi_{n}\left|f(x)-\widehat{f}_{N}(x)\right| & \leq \chi_{n}\left|f(x)-f_{N}(x)\right|+\chi_{n}\left|\left\langle h^{N}(x), b_{N}-\widehat{b}_{N}\right\rangle\right| \\
& \leq O\left(N^{-\alpha}\right)+\chi_{n}\left\|h^{N}(x)\right\| \cdot\left\|b_{N}-\widehat{b}_{N}\right\|
\end{aligned}
$$

for $x \in A$, and according to (2) we further obtain

$$
\chi_{n}\left\|f-\widehat{f}_{N}\right\|_{\infty} \leq O\left(N^{-\alpha}\right)+\chi_{n}\left\|b_{N}-\widehat{b}_{N}\right\| M_{N} c^{-1 / 2} .
$$

Since the condition $N(n) M_{N(n)}^{2} / n \rightarrow 0$ as $n \rightarrow \infty$ implies $P\left(\chi_{n} \neq 1\right) \rightarrow 0$ we get the assertion by Lemma 2.2.

The uniform convergence rates for the above series estimators do not attain Stone's [12] bound on the best obtainable rate for the uniform error but they improve on some rates obtained earlier, e.g. on those of Cox [2].

4. Conclusions. As proved in [8], if we use orthogonal systems of analytic functions to construct estimators of regression functions (e.g. trigonometric functions or multivariate polynomials), the normal equations matrix 
$G_{n}$ is almost surely positive definite for $N \leq n$ for any density $\varrho$. Thus, in that case the estimators are uniquely defined with probability one.

Let $\beta=\left(\beta_{1}, \ldots, \beta_{d}\right)$ be a vector of nonnegative integers, and $|\beta|=$ $\sum_{k=1}^{d} \beta_{k}$. For a vector function $f(x)=\left(f_{1}(x), \ldots, f_{N}(x)\right)^{T}$ define the vector of partial derivatives $\partial^{|\beta|} f(x)=\partial^{|\beta|} f(x) / \partial x_{1}^{\beta_{1}} \ldots \partial x_{d}^{\beta_{d}}$ and for any nonnegative integer $r$ let $\|f\|_{r}=\max _{|\beta| \leq r} \sup _{x \in A}\left\|\partial^{|\beta|} f(x)\right\|$.

Now put $P_{r}(N)=\max _{|\beta| \leq r} \sup _{x \in A}\left\|\partial^{|\beta|} e^{N}(x)\right\|$ and assume that $\varrho \in$ $L^{1}(A), \varrho \geq c>0$, and for $N>0$ there exists a vector $b_{N}$ such that $\left\|f-b_{N}^{T} e^{N}\right\|_{r} i=O\left(N^{-\alpha}\right)$, where $\alpha>0$. Under the above assumptions, using the same technique as in the proof of Theorem 3.1 we can prove that $\left\|f-\widehat{f}_{N(n)}\right\|_{r}=O_{p}\left(P_{r}(N(n))\left(N(n)^{1 / 2} / n^{1 / 2}+N(n)^{-\alpha}\right)\right)$ if the natural numbers $N(n)$ satisfy $\lim _{n \rightarrow \infty} N(n)=\infty$ and $\lim _{n \rightarrow \infty} N(n) P_{0}^{2}(N(n)) / n=0$.

The exponent $\alpha$ defining the decrease rate of the uniform approximation error of the regression function and its derivatives up to order $r$ is related not only to the smoothness of the regression function but also to the dimensionality of $X$ and the size of $r$. For example, if $f$ is continuously differentiable of order $s$ on $[-1,1]^{d}$, then in the case of polynomial approximation and $r=0$ we have $\alpha=s / d$ according to Lorentz [5]. It is much more difficult to find in the literature a corresponding result for the case when $r>0$, except in two cases. Namely, when $X$ is univariate it is well known that $\alpha=s-r$ (see [5]), and when $f$ is analytic it is known that for any $r$ the assumption of uniform approximation rate in the norm $\|*\|_{r}$ will hold with $\alpha$ equal to an arbitrarily large positive number.

Now consider the observation model $Y_{i}=f\left(X_{i}\right)+\eta_{i}, i=1, \ldots, n$, where the $\eta_{i}$ are realizations of some strictly stationary $\beta$ mixing process, centered in expectation, with $\beta$ mixing sequence $\left(\beta_{k}\right)_{k \geq 0}$ satisfying the condition $\sum_{k \geq 0} \beta_{k}<\infty$ (see [13]). In that case in view of the inequalities $\chi_{n} \lambda_{n}^{-1} \leq 2$ and $\left\|h_{N}\right\|_{\infty} \leq M_{N} / \sqrt{c}$ we have

$$
\begin{aligned}
E \chi_{n} \| H_{n}^{-1} & \frac{1}{n} \sum_{i=1}^{n} \eta_{i} h^{N}\left(X_{i}\right)\left\|^{2} \leq E_{X} E_{\eta} \chi_{n} \lambda_{n}^{-2}\right\| \frac{1}{n} \sum_{i=1}^{n} \eta_{i} h^{N}\left(X_{i}\right) \|^{2} \\
& \leq 4 E_{X} \chi_{n} \frac{1}{n^{2}} \sum_{i=1}^{n} \sum_{j=1}^{n} E_{\eta} \eta_{i} \eta_{j}\left\langle h^{N}\left(X_{i}\right), h^{N}\left(X_{j}\right)\right\rangle \\
& \leq 4 E_{X} \chi_{n} \frac{1}{n^{2}} \sum_{i=1}^{n} \sum_{j=1}^{n}\left|\operatorname{cov}\left(\eta_{i}, \eta_{j}\right)\right| \cdot\left\|h^{N}\left(X_{i}\right)\right\| \cdot\left\|h^{N}\left(X_{j}\right)\right\| \\
& \leq \frac{4 M_{N}^{2}}{n^{2} c} \sum_{i=1}^{n} \sum_{j=1}^{n}\left|\operatorname{cov}\left(\eta_{i}, \eta_{j}\right)\right| \leq \frac{8 M_{N}^{2}}{n c} \sum_{i=1}^{n}\left|\operatorname{cov}\left(\eta_{i}, \eta_{1}\right)\right|=O\left(\frac{M_{N}^{2}}{n}\right),
\end{aligned}
$$

since for $\beta$ mixing processes satisfying the imposed conditions the sums 
$\sum_{i=1}^{n}\left|\operatorname{cov}\left(\eta_{i}, \eta_{1}\right)\right|$ are bounded by a constant (see Theorem 2.2 in [13] for details). Thus, for such observation models we obtain, as in Lemma 2.2, the bound $E \chi_{n}\left\|\widehat{b}_{N}-b_{N}\right\|^{2}=O\left(M_{N}^{2} / n+N^{-2 \alpha}\right)$, which implies that for orthogonal systems satisfying (5) the convergence rates of the relevant errors are the same as in the case of independent observations.

\section{References}

[1] L. Birgé and P. Massart, Minimum contrast estimators on sieves: exponential bounds and rates of convergence, J. Bernoulli Soc. 4 (1998), 329-375.

[2] D. D. Cox, Approximation of least squares regression on nested subspaces, Ann. Statist. 16 (1988), 713-732.

[3] L. Györfi and H. Walk, On the strong universal consistency of a series type regression estimate, Math. Methods Statist. 5 (1996), 332-342.

[4] J. Z. Huang, Projection estimation in multiple regression with application to functional ANOVA models, Ann. Statist. 26 (1998), 242-272.

[5] G. G. Lorentz, Approximation of Functions, Holt, Reinehart \& Winston, New York, 1966.

[6] G. Lugosi and K. Zeger, Nonparametric estimation via empirical risk minimization, IEEE Trans. Inform. Theory IT-41 (3) (1995), 677-687.

[7] P. Niyogi and F. Girosi, Generalization bounds for function approximation from scattered noisy data, Adv. Comput. Math. 10 (1999), 51-80.

[8] W. Popiński, On least squares estimation of Fourier coefficients, and of the regression function, Appl. Math. (Warsaw) 22 (1993), 91-102.

[9] - Consistency of trigonometric and polynomial regression estimators, ibid. 25 (1998), 73-83.

[10] -, A note on orthogonal series regression function estimators, ibid. 26 (1999), 281-291.

[11] E. Rafajł owicz, Nonparametric least-squares estimation of a regression function, Statistics 19 (1988), 349-358.

[12] C. J. Stone, Optimal global rates of convergence for nonparametric regression, Ann. Statist. 10 (1982), 1040-1053.

[13] G. Viennet, Least-square estimation for regression on random design for absolutely regular observations, Statist. Probab. Lett. 43 (1999), 13-23.

Waldemar Popiński

Department of Survey Organization

Central Statistical Office

Al. Niepodległości 208

00-925 Warszawa, Poland

E-mail: w.popinski@stat.gov.pl 